\title{
Differentially expressed microRNAs and their target genes in the hearts of streptozotocin-induced diabetic mice
}

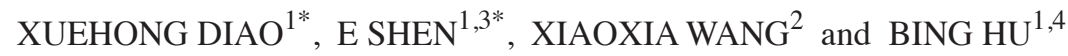 \\ ${ }^{1}$ Departments of Ultrasound in Medicine and ${ }^{2}$ Nephrology, Shanghai Jiaotong University Affiliated 6th People's Hospital; \\ ${ }^{3}$ Cardiovascular Disease Lab, and ${ }^{4}$ Shanghai Institute of Ultrasound in Medicine, Shanghai 200233, P. R. China
}

Received January 20, 2011; Accepted April 21, 2011

DOI: $10.3892 / \mathrm{mmr} .2011 .489$

\begin{abstract}
Persistent hyperglycemia in diabetic patients has been associated with cardiac hypertrophy, myocardial fibrosis and cardiac dysfunction. However, the underlying mechanisms of this association have yet to be fully elucidated. The aim of this study was to investigate the expression and function of microRNAs (miRNAs) in diabetic cardiomyopathy. miRNA expression profiles were examined by miRNA microarray analysis in heart tissue from streptozotocin (STZ)-induced diabetic mice and non-diabetic mice. The targets of the altered miRNAs were predicted using the Sanger database. Then, the targets RASA1, RAC1, TGFB3 and COL1A1, related to cardiac hypertrophy or myocardial fibrosis, were selected to analyze the miRNA level by real-time reverse transcription (RT-PCR). Gene Ontology (GO) was further applied to describe the function of each miRNA target gene and to elucidate their combined effects in diabetic cardiomyopathy. Up-regulated $(n=10)$ and down-regulated $(n=6)$ miRNAs were identified in diabetic cardiomyopathy. Target genes $(n=192)$ were pooled from the Sanger database. Among the 192 targets, the mRNA expression of RASA1, RAC1, TGFB3 and COL1A1 was increased in diabetic cardiomyopathy. Thirty one GO functions were enriched in diabetic cardiomyopathy. These results demonstrate that miRNAs may mediate cardiac hypertrophy and myocardial fibrosis in diabetic cardiomyopathy via their targets, and provide insights into the pathogenesis of diabetic cardiomyopathy.
\end{abstract}

\section{Introduction}

Diabetic cardiomyopathy is a myocardial disease caused by diabetes mellitus independent of hypertension and coronary

Correspondence to: Dr Bing Hu and Dr E Shen, Department of Ultrasound in Medicine and Cardiovascular Disease Lab, Shanghai Jiaotong University Affiliated 6th People's Hospital, Shanghai Institute of Ultrasound in Medicine, Shanghai 200233, P.R. China E-mail: binghuzz@gmail.com; shene1001@hotmail.com

* Contributed equally

Key words: micro ribonucleic acid molecules, diabetic cardiomyopathy, microarray, target genes artery disease. Clinical and experimental studies have revealed that diabetic cardiomyopathy is characterized by myocardial hypertrophy, prominent interstitial fibrosis and cardiac dysfunction. These cardiac structural and functional changes can ultimately lead to heart failure and even sudden death $(1,2)$. Therefore, elucidation of the molecular mechanisms underlying hyperglycemia is essential for the development of effective novel strategies against diabetic cardiomyopathy.

microRNAs (miRNAs) are a class of highly conserved, small non-coding RNAs that transcriptionally regulate gene expression (3). miRNA expression is tightly controlled in a tissue-specific and developmental stage-specific manner. Increasing evidence indicates that miRNAs play an important gene-regulatory role in heart development, vascular angiogenesis and various aspects of cardiac disease, such as ischemic myocardial injury, arrhythmia, cardiac fibrosis and heart failure (4-6). In particular, many studies on specific miRNAs in animal models and human hearts have demonstrated the essential roles of miRNAs in cardiac hypertrophy and glucose homeostasis (7-9). Given that miRNAs participate in such fundamental processes, further investigation of their potential role in diabetic cardiomyopathy is warranted. In the present study, the expression profiles of the miRNAs were determined by microarray analysis, and then the target genes of the miRNAs were predicted using the Sanger database. Among the predicted target genes, the mRNA levels of representative genes related to cardiac hypertrophy or myocardial fibrosis were validated using real-time RT-PCR. Furthermore, the function of the altered miRNAs was analyzed by Gene Ontology (GO).

\section{Materials and methods}

Induction of diabetes. All experimental procedures were performed according to the Guidelines for the Care and Use of Laboratory Animals published by the US National Institutes of Health (NIH publication no. 85-23, revised 1996) and were approved by the Animal Use and Care Committee of Shanghai Jiaotong University School of Medicine.

Streptozotocin (STZ) destroys pancreatic $\beta$ cells and produces severe insulin deficiency and hyperglycemia, leading to type I diabetes. Adult C57BL/6 male mice (8 weeks old; 23-25 g) were intraperitoneally injected with a single dose of STZ at $150 \mathrm{mg} / \mathrm{kg}$. At $72 \mathrm{~h}$ after STZ injection, the whole blood glucose levels were measured using the Optium Xceed ${ }^{\mathrm{TM}}$ 
Table I. Oligonucleotide sequences for RT-PCR.

\begin{tabular}{lll}
\hline Gene & GenBank no. & Sequence \\
\hline ANP & NM_008725.2 & TGACAGGATTGGAGCCCAGAGAGCTGCGTGACACACCACAAG \\
BNP & NM_008726.4 & ATCGGATCCGTCAGTCGTTTGCCAGGCAGAGTCAGAAACTGGAG \\
$\alpha-$ MHC & NM_010856.3 & TGGTGGACAAGCTACAGTTGAAGGCGGAACTTGGACAGGTTGGTG \\
$\beta$-MHC & NM_080728.2 & TAACCCGAGGCAAGCTCACACACAATCATGCCGTGCTGAC \\
RASA1 & NM_145452.3 & TCAGGTAGCAGCCTGTGTTCCTGGAATGTGGGAAAGTGTG \\
RAC1 & NM_009007.2 & CCCAATACTCCTATCATCCTCGCAGCAGGCATTTTCTCTTCC \\
TGFB3 & NM_009368.3 & GGAAATCAAATTCAAAGGAGGTGGAGTTGGCATAGTAACCCTTAGG \\
COL1A1 & NM_007742.3 & CCTGCCTGCTTCGTGTAAACTTTGG GTTGTTCGTCTGTTTCC \\
GAPDH & NM_008084.2 & TGTGTCCGTCGTGGATCTGATTGCTGTTGAAGTCGCAGGAG \\
\hline
\end{tabular}

Table II. The LVHW/BW ratio and cardiac function in experimental mice.

\begin{tabular}{lcc}
\hline Parameters & $\begin{array}{c}\text { Control group } \\
(\mathrm{n}=10)\end{array}$ & $\begin{array}{c}\text { STZ } \\
(\mathrm{n}=15)\end{array}$ \\
\hline Blood glucose (mmol/l) & $24.0 \pm 3.0$ & $8.3 \pm 0.6$ \\
Body weight (g) & $27.2 \pm 1.6^{\mathrm{a}}$ & $19.7 \pm 1.8^{\mathrm{a}}$ \\
LVHW/BW Ratio (mg/g) & $3.75 \pm 0.07$ & $4.61 \pm 0.10^{\mathrm{a}}$ \\
EF (\%) & $92.32 \pm 2.87$ & $54.70 \pm 4.92^{\mathrm{a}}$ \\
Fs (\%) & $65.42 \pm 1.77$ & $28.56 \pm 2.06^{\mathrm{a}}$ \\
\hline
\end{tabular}

${ }^{\mathrm{a}} \mathrm{p}<0.01$ vs. Control group. LVHW/BW, left ventricular heart weight to body weight ratio

diabetes monitoring system (USA). Mice with blood glucose levels $\geq 18.6 \mathrm{mmol} / 1$ were used in the experimental group, while citrate buffer-treated mice were used as the control. All mice were housed in a pathogen-free 'barrier' facility.

Echocardiographic measurements. After 8 weeks of diabetes, cardiac function was evaluated by echocardiography on conscious mice using an HP SONOS 5500 ultrasound system equipped with a 14-MHz transducer. Briefly, two-dimensional images were obtained of a parasternal short-axis view, guided with M-mode at the midventricular level in this plain. Left ventricle (LV) internal diameters and wall thickness were measured at the end diastole and end systole (at least 3 cardiac cycles), after which LV ejection fraction (EF) and fractional shortening (FS) were calculated.

Histological analysis. The mice were sacrificed one day after echocardiographic examination. The excised hearts were rinsed in PBS buffer and weighed to calculate the ratio of heart weight to body weight (HW/BW). Histological analysis of heart tissues was performed according to standard procedures. Briefly, LV samples were fixed in $4 \%$ paraformaldehyde for $12 \mathrm{~h}$ at room temperature and embedded in paraffin. Subsequently, 4- $\mu$ m-thick sections were stained with haematoxylin and eosin (H\&E) for measurement of cardiomyocyte size using NIH ImageJ software. Approximately 100 randomly selected cardiomyocytes from each group $(n=5)$ were analyzed to measure the cross-sectional cardiomyocyte area. Myocardial interstitial fibrosis was determined by Masson's trichrome staining. The tissue was imaged, digital images were copied into the NIH ImageJ software, and the threshold was set to separate blue staining collagen from red staining muscle tissues. The area of blue staining served as a general indicator for fibrosis. From each group $(n=5), 30-50$ randomly selected frames were analyzed. Histological evaluations were performed by two individuals in a blinded manner and the results were averaged.

Real-time RT-PCR. Total RNA was isolated from LV samples using TRIzol reagent (Invitrogen) according to the manufacturer's protocol. Total RNA $(2 \mu \mathrm{g})$ was subjected to reverse transcriptase reaction to synthesize the cDNA using the ExScript RT reagent kit (Takara, Kusatsu, Japan). Total primer sequences are listed in Table I. Glyceraldehyde 3-phosphate dehydrogenase (GAPDH) as the internal control was also amplified under the same conditions to normalize reactions. The relative fold change was calculated based on the $2^{\Delta \Delta} \mathrm{CT}$ method.

microRNA assay. miRNAs were enriched from total RNA using the mirVana miRNA isolation kit (Ambion, Austin, TX, USA) and labeled with the mirVana array labeling kit (Ambion) according to the manufacturer's protocol. The miRNA microarray assay was performed using the CapitalBio mammalian miRNA array V4.0 (CapitalBio Corporation, Beijing, China) containing 1320 probes including human, mouse and rat mature miRNAs. The differentially expressed miRNAs were selected using significance analysis of microarray (SAM) software 3.02. Criteria for selection were set as a false discovery rate (FDR) of $<5 \%$ and a fold change of $>2$.

Individual miRNA expression was confirmed using individual miRNA primers specific for reverse transcription (RT). RT-PCR reactions followed the protocol of the SYBR ${ }^{\circledR}$ Green PCR Master Mix (Applied Biosystems). 
A

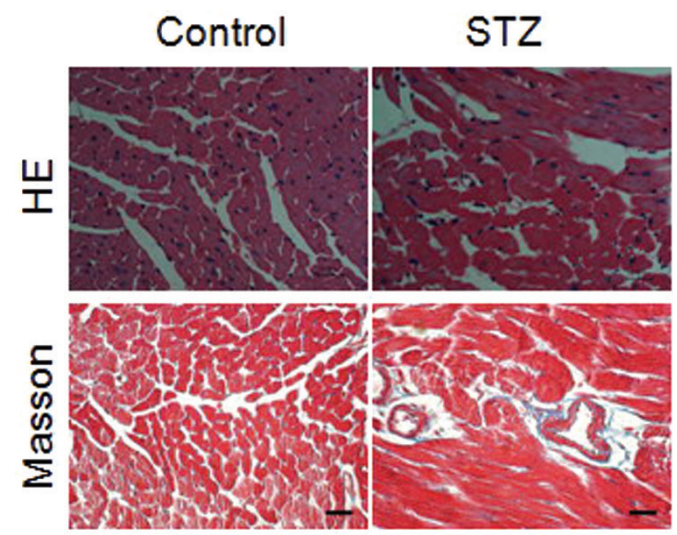

B

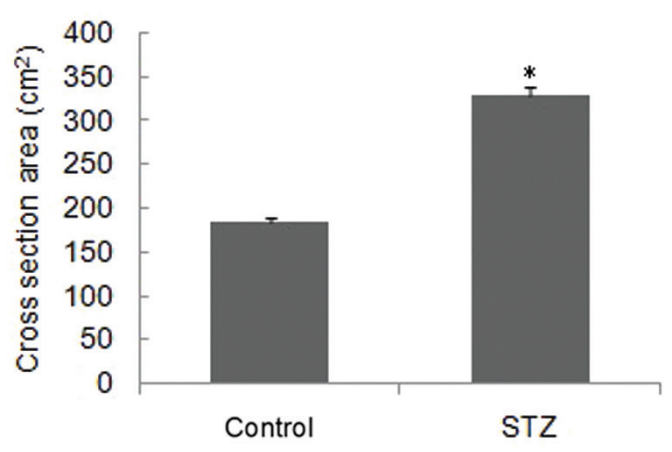

C
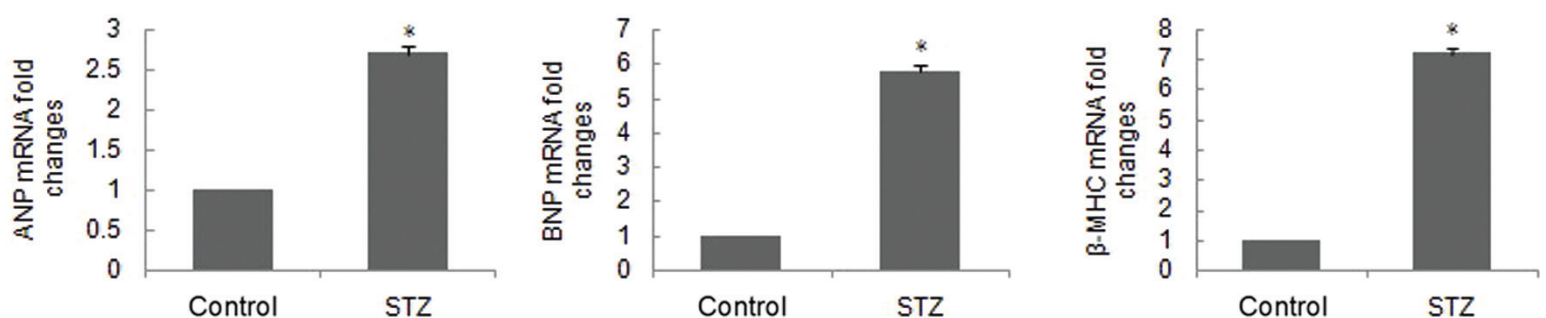

Figure 1. Diabetic hearts exhibited cardiac hypertrophy and extensive interstitial fibrosis. (A) Cardiac myocyte size was dramatically increased in the diabetic hearts as revealed by H\&E staining (top panel). Myocardial interstitial fibrosis was evaluated by Masson's trichrome staining (bottom panels). Fibrosis of the diabetic heart was noted, in particular an area of extensive fibrosis in the tissue surrounding blood vessels. Magnification, $\mathrm{x} 40$. Bar, $50 \mu \mathrm{m}$. (B) Quantitative data from a cross-sectional area of cardiomyocytes. Results are presented as the mean $\pm \mathrm{SD}, \mathrm{n}=5$. ${ }^{*} \mathrm{P}<0.05$ vs. the control. (C) Expression of ANP, BNP and $\beta$-MHC in experimental mouse hearts was measured by real-time RT-PCR. Data are the mean \pm SD of values from 5 hearts. " $\mathrm{P}<0.05$ vs. the control was observed.
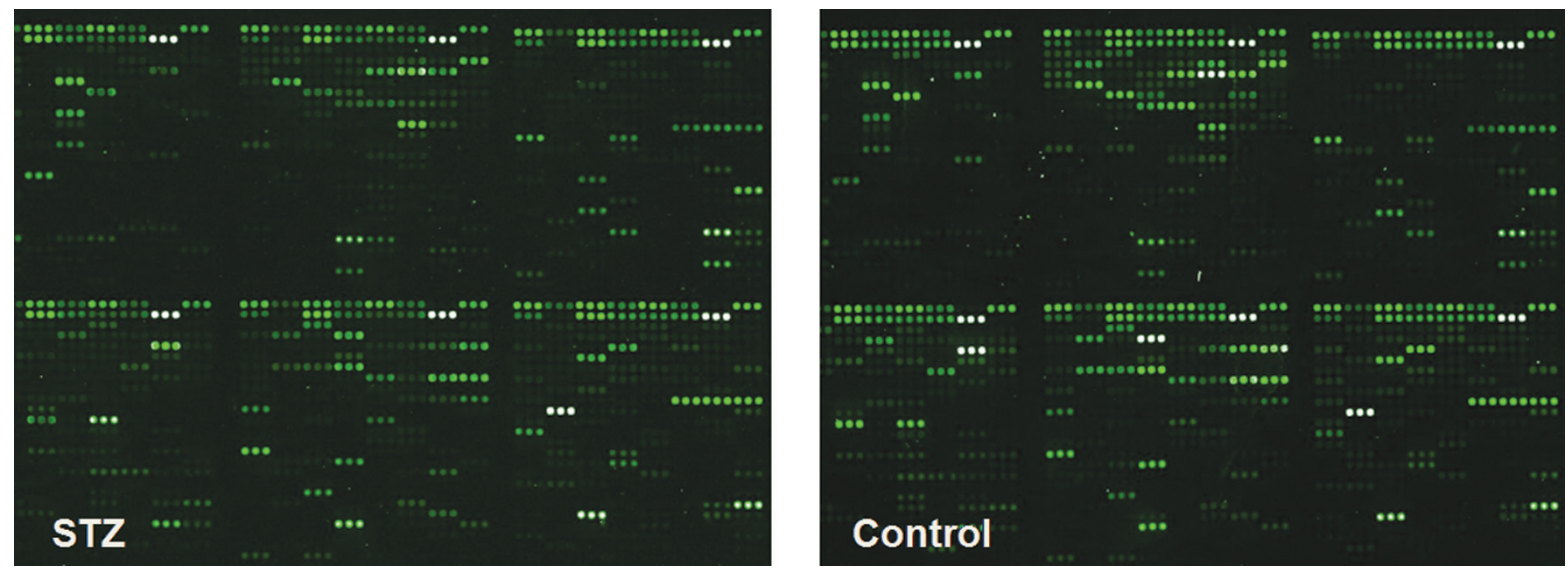

Figure 2. miRNA expression patterns in diabetic hearts. Representative images from two chips from a diabetic and a non-diabetic heart. Differences in the distribution of fluorescence intensities represent the differentially expressed microRNAs from the two chips. The sample was labeled with Cy3 fluorescent dyes in pseudocolor. STZ, hybridization signal of sample STZ; control, hybridization signal of the control sample.

microRNA target gene analysis. miRNA target genes were pooled from the Sanger database (http://microrna.sanger. ac.uk/). This prediction algorithm is a commonly used method for the accurate identification of miRNA targets. The top $25 \%$ of miRNA targets that had been assigned the highest numbers of miRNA interaction sites were collected.

Analysis of microRNA target gene functions. GO provides the molecular function, biological process and cellular compo- nents of gene products (10), which were used to annotate the functions of the miRNA targets. Within the significant category, the enrichment Re was expressed as: $\operatorname{Re}=\left(n_{\mathrm{f}} / \mathrm{n}\right) /\left(\mathrm{N}_{\mathrm{f}} / \mathrm{N}\right)$, where $n_{f}$ is the number of differentially expressed genes within the particular category, $n$ is the total number of genes within the same category, $\mathrm{N}_{\mathrm{f}}$ is the number of differentially expressed genes in the entire microarray, and $\mathrm{N}$ is the total number of genes in the microarray. The two-sided Fisher's exact test and $\chi^{2}$ test were used to classify the GO category, 
Table III. Target genes of identified miRNAs in diabetic hearts.

miRNA Putative target

miR-195

FGF2, MAP3K3, MAP2K1, MAP3K4, BCL2, ACVR2A, BTRC, CDC42, CCND1, ZYX, CDC25A, FGF7, ITGA2, ADRB2, SMAD5, LRP6, CRKL, SMAD3, BAI1, AXIN2, ACOX1, TRIP10, WNT7A, LAMC1, SIAH1, EIF4E, FZD10, RELN, PPP2R1A, MRAS, GRIN1, RAF1, BMPR1A, SMAD7, ENAH, CX3CL1

miR-208

NLK, MAP4K3, CSNK2A2, ACVR1

miR-1

RASA1, IGF1, GJA1, MAPK3, MAP4K3, SMAD4, VEGFA, SFRP1, MET, EIF4E, CDC14A, BAIAP2, DLG1, FZD7, CTBP2 PREX1, DAAM1, PTPN1, YWHAB, PDGFA, RAPGEF2

miR-21

PPARA, ACVR2A, PITX2, FASLG, SMAD7, CDC25A, RASA1, PPP3CA, ARHGEF7, RASGRP1, BCL2

miR-24

ACVR1B, RASGRP4, TCF7, VAV1, RAP1A, RASA1, FLT1, PDGFRA, ATP2A2, SSH2, RAP1B, CDKN1B, ITGA3, NLK, COL11A2, PTPRF

miR-199a-3p

UQCRB, ITGB8, ERBB4, ACVR2B, ACVR2A, SCD, MAP3K4, ACOX1, FGF7, MAP3K5, NLK, RB1, FBXW11, TMSB4X, TMSL3, ACVR1C, PTPRF, ITGA3, CDC14A, PDGFRA, YWHAE, DDIT4, FN1, CHAD, ITGA6, ARHGEF12, IQGAP2, PIK3R1, IGF1, PXN, CAV2

miR-142-3p

ITGB8, RAC1, ITGAV, APC, ROCK2, TGFBR1, GNAQ, MYH10, RHEB, MAP3K11, CRK, ACVR2A, FOXO1, PPP3R1, PPP3CA, MYH9, ARHGEF12, TIRAP, PMAIP1

$\operatorname{miR}-373$

PFN2, NF1, MAP3K14, LEF1, MAP3K7, TNXB, MAP3K11, LEFTY1, TAOK2, MAPK9, RPS6KA1, ITGB8, CCND1, PPP3R1, RPS6KA5, CRK, ULK1, SMAD2, FLT1, MEF2C

miR-20a PPARA, TRIP10, SMAD5, HIF1A, SCD5

miR-29a

TGFB3, COL1A1, COL3A1, COL1A2, COL5A2, PRKG1, COL2A1, SLK, ITGA11, TNFRSF1A, CSNK1A1, COL6A3, CX3CL1, COL5A3, CAV2, PTEN, ITGA6, PDGFC, ABL1, PPP1R3D, LAMC1

miR-700

CHRM2, RAF1, ILK, CTBP2, LRDD, CTBP1, RAC1, CYP27A1, TNNC1, PTK2B, HK1, GNA11, ACVRL1, HK3, NKD2, CREBBP, ITGA11, RASGRF1, ANGPTL4, HSPA1L, SRF, HIF1A, HSPA2, AKT1, PPP2CA, PFN1, CCL3, CD40

miR-705

FGF7, COL1A1, PARVA, CCL5

miR-143

SSH2, KRAS, TPM3, BCL2, LIMK1, PDGFRA, MAP3K7, ITGA6, COL5A3, FGF1, COL5A2, RB1

miR-220b

CX3CR1, ACVR1B, IGFBP3, NLK

miR-221

CDKN1B, RAP1B, WNT1, SFRP2, CD4, PAK1, FLOT2, ANGPTL2, PTEN, NLK, STK4

miR-499-3p

NTRK2, FOXO1, CHP, PDPK1, MAP4K3, GNG2, ELK4, UQCRB, BTRC, RAPGEF2, FZD9

and the FDR was calculated to correct the P-value. A P-value $<0.01$ and an FDR $<0.01$ were used as the threshold to select significant GO categories.

Statistical analysis. Data are presented as the mean \pm SD. Differences between two groups were compared using the unpaired Student's t-test. A value of $\mathrm{P}<0.05$ was considered statistically significant.

\section{Results}

Characterization of diabetic animals. At $72 \mathrm{~h}$ after STZ injection, diabetes was confirmed in mice with blood glucose levels $\geq 18.6 \mathrm{mmol} / 1$. Throughout the 8 -week study period, STZ-induced diabetic mice displayed severe hyperglycemia. This was associated with a decrease in body weight, resulting in a significantly higher $\mathrm{HW} / \mathrm{BW}$ ratio as compared to the non-diabetic controls. LV EF and fractional shortening (FS) were significantly reduced in the diabetic hearts (Table II). H\&E staining revealed the appearance of enlarged myocardial cells with large and irregular nuclei in the diabetic hearts. The cardiomyocyte cross-sectional area was significantly increased in the STZ mouse hearts. Moreover, increased blue staining was observed in STZ-treated hearts, particularly in the perivascular area. By contrast, no blue dye was found in the non-diabetic hearts (Fig. 1). ANP, BNP and $\beta$-MHC, 

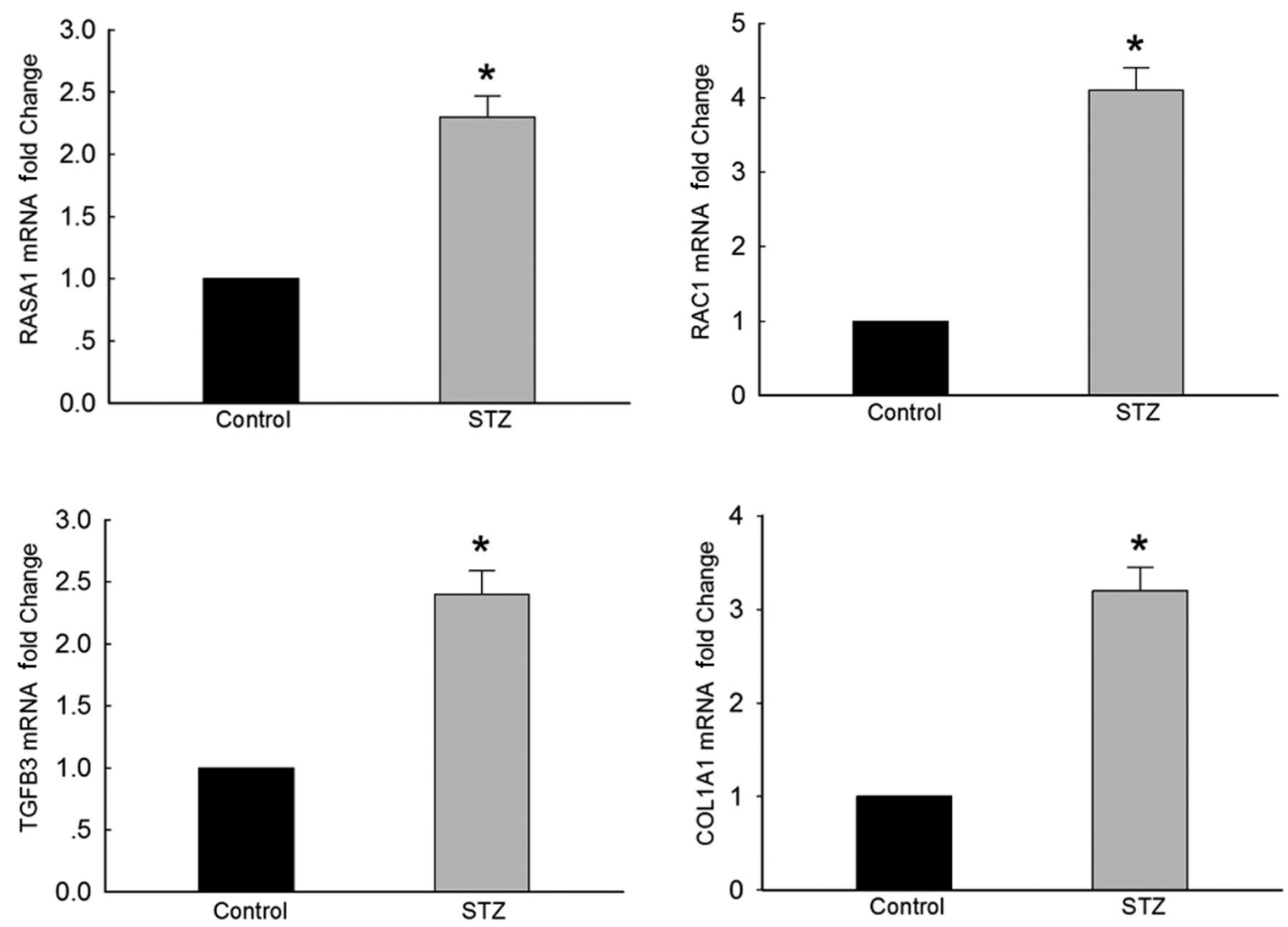

Figure 3. High mRNA expression levels of RASA1, RAC1, TGFB3 and COL1A1 in experimental mouse hearts. Gene expression levels are presented as the fold change (relative to the control). ${ }^{*} \mathrm{P}<0.05$ vs. the control.

markers of cardiac hypertrophy, were expressed at high levels in the diabetic hearts.

Up or down-regulation of miRNA in STZ-induced diabetic hearts. To investigate the potential involvement of miRNAs in diabetic cardiomyopathy, hearts from STZ-induced mice $(n=3)$ and non-diabetic hearts $(n=3)$ were examined using miRNA microarray analysis. Compared to the control hearts, the expression of 19 miRNAs was altered in the STZ-induced diabetic hearts, of which 16 miRNAs were further verified by real-time RT-PCR. Differentially expressed miRNA patterns in the diabetic hearts were observed using the microarray chip (Fig. 2). Expression of 10 miRNAs (miR-195, miR199a-3p, miR-700, miR-142-3p, miR-24, miR-21, miR-221, miR-499-3p, miR-208 and miR-705) was up-regulated, whereas the expression of the remaining 6 miRNAs (miR29a, miR-1, miR-373, miR-143, miR-20a and miR-220b) was down-regulated.

Target gene prediction. Using the Sanger database, 192 target genes of the 16 differentially epxressed miRNAs were collected. Among these were 36 of miR-195 target genes, including FGF2, MAP3K3, MAP2K1, MAP3K4, BCL2, ACVR2A, BTRC, CDC42, CCND1, ZYX, CDC25A, FGF7, ITGA2, ADRB2, SMAD5, LRP6, CRKL, SMAD3, BAI1, AXIN2, ACOX1, TRIP10, WNT7A, LAMC1, SIAH1, EIF4E, FZD10, RELN, PPP2R1A, MRAS, GRIN1, RAF1, BMPR1A, SMAD7, ENAH and CX3CL1. MiR-199a-3p had 31 associated target genes, including UQCRB, ITGB8, ERBB4, ACVR2B,
ACVR2A, SCD, MAP3K4, ACOX1, FGF7, MAP3K5, NLK, RB1, FBXW11, TMSB4X, TMSL3, ACVR1C, PTPRF, ITGA3, CDC14A, PDGFRA, YWHAE, DDIT4, FN1, CHAD, ITGA6, ARHGEF12, IQGAP2, PIK3R1, IGF1, PXN, CAV2. MiR-700 contained 28 putative target genes. The details of the differentially expressed miRNA target genes are listed in Table III.

Cardiac hypertrophy- or myocardial fibrosis-related target gene expression in diabetic hearts. Among the 192 target genes, RASA1, RAC1, TGFB3 and COL1A1, related to cardiac hypertrophy or myocardial fibrosis, respectively, were selected for analysis of mRNA expression levels by real-time RT-PCR. The results indicated that RASA1 and RAC1, related to cardiac hypertrophy, or TGFB3 and COL1A1, related to myocardial fibrosis, were significantly increased in the heart tissue of diabetic mice (Fig. 3).

Gene Ontology analysis of target gene function. GO analysis, based on these 192 targets, revealed that 30 GO terms were involved in diabetic cardiomyopathy. The five high-enrichment GOs were insulin-like growth factor receptor signaling, the JNK cascade, leukocyte adhesive activation, transmembrane receptor protein serine/threonine kinase signaling, the MAPKKK cascade and collagen fibril organization (Fig. 4). The different degrees of the other 26 enrichment GOs are listed in Fig. 4. The results indicate that miRNAs preferentially targeted genes involved in a wide spectrum of regulatory functions and select biological processes in the diabetic heart. 


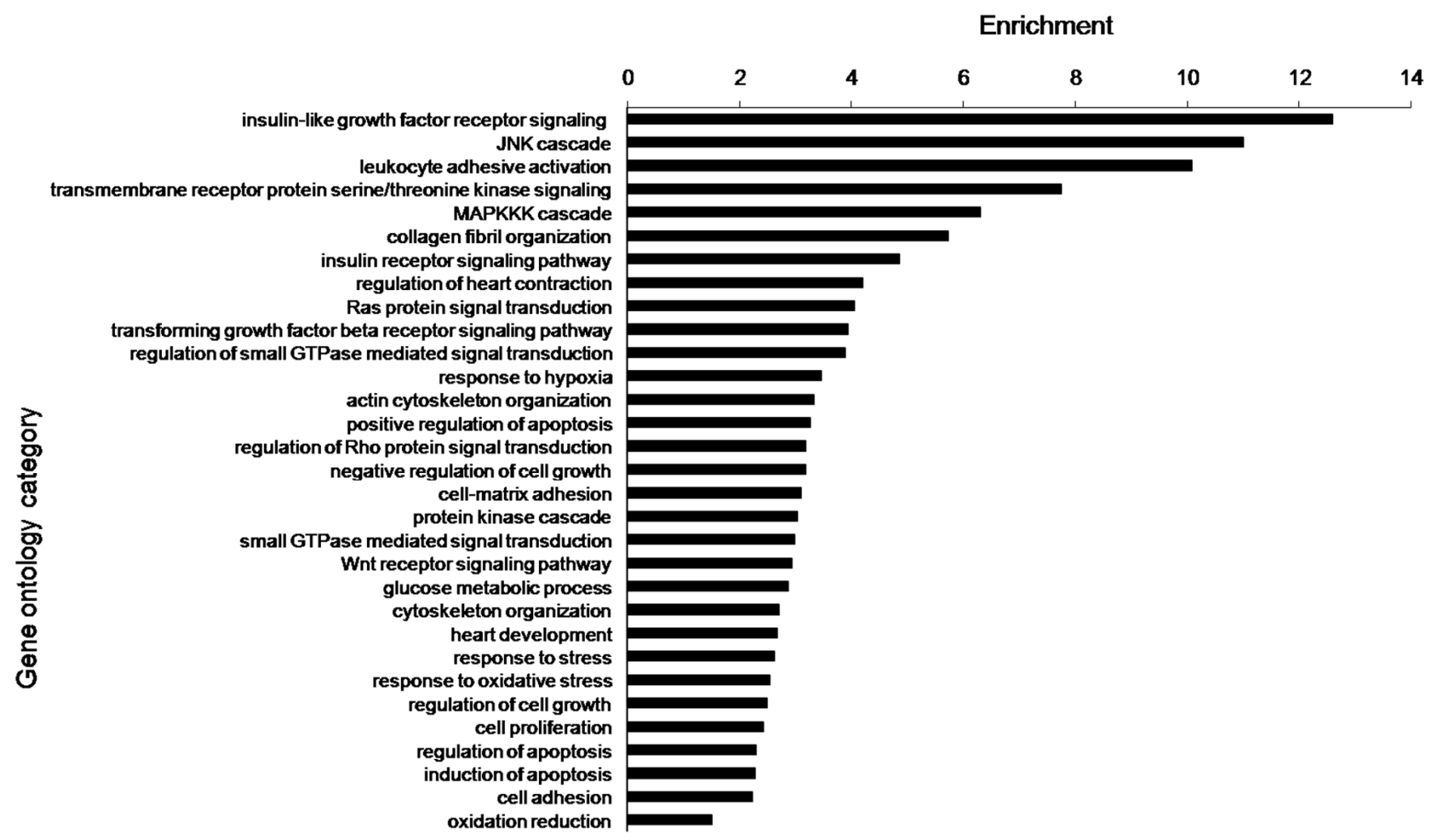

Figure 4. GO category based on biological process for differentially expressed genes. Vertical axis, GO category; horizontal axis, enrichment of GO.

\section{Discussion}

miRNAs belong to a class of short non-coding RNAs known to suppress the expression of protein-coding genes through imperfect base-pairing with the 3'-untranslated region (3'UTR) of the target mRNA. It has been reported that cardiac miRNAs may play a major role in heart development and function (11). One previous study also found that specific miRNAs play an essential regulatory role in hyperglycemiainduced cardiac hypertrophy (12). However, miRNAs have yet to be detected and very little is known regarding miRNA expression levels or patterns in diabetic cardiomyopathy.

In this study, the expression of ten miRNAs was increased, while that of six miRNAs was decreased, in STZ-treated mouse hearts. Among the differentially expressed miRNAs, miR-1, miR-208a and miR-195 were recently recognized to be critical in the pathological process of heart disease. An in vitro study demonstrated that over-expression of miR-1 decreased the size of cardiac myocytes and the hallmarks of hypertrophy (13). In the present study, miR-1 was dramatically down-regulated in diabetic hearts with impaired cardiac function, increased cardiac hypertrophy and myocardial fibrosis, which are hypothesized to be critical in determining cardiomyocyte hypertrophy or cardiac fibrosis. Up-regulation of miR-208 and miR-195 was also observed in diabetic hearts in the present study. It was reported that miR-208 is specifically expressed in heart tissue and is required for cardiomyocyte hypertrophy or myocardial fibrosis (14). miR-195 may play an active role in morphological changes in cardiomyocytes and pathological cardiac remod- eling. Cardiac over-expression of miR-195 increased the left ventricular wall thickness and induced cardiac dysfunction and heart failure in mice (15). Our data indicate that miR-208 and miR-195 may contribute to structural or functional changes in the diabetic heart. Moreover, miR-21 and miR-24 are key regulators of cardiac hypertrophy (15-17), whereas miR-199a-3p is up-regulated in rats with myocardial infarction (18). Our results revealed that expression of miR-21, miR-24 and miR-199a-3p was increased in mice with cardiac hypertrophy induced by hyperglycemia, which suggests that these miRNA may be involved in the pathological process in the diabetic heart. Other miRNA, including miR-142-3p, miR-373, miRNA-20, miRNA29a, miR-700, miR-499-3p, miR-705, miR-143, miR-220b and miR-221, have not been investigated in heart disease. Further investigations focusing on these miRNAs are required to determine their roles in the pathogenesis of the diabetic heart.

In order to explore the targets of the differentially expressed miRNAs, the Sanger database was consulted. A total of 192 target genes of the 16 differentially expressed miRNAs were identified. Our results indicate that each single miRNA regulates multiple target genes. For example, miR-195 and miR-199a-3p have over 30 predicted target genes. In addition, a gene may be regulated by multiple miRNAs. Among these target genes, NLK, regulated by five miRNAs (miR-208, miR-24, miR-221, miR-220b and miR-199a-3p), was particularly prominent in the hearts of the diabetic mice. ACVR2A was targeted by four miRNAs including miR-21, miR-195, miR-199a-3p and miR-142-3p. MAP4K3, FGF7, BCL2, RASA1, ITGB8, ITGA6 and PDGFRA were regulated 
by three different miRNAs, respectively. These findings suggest that miRNAs act in a complex functional network by specifically regulating target genes. In the present study, histological analysis revealed that the hearts of the diabetic mice were characterized by cardiac hypertrophy and myocardial fibrosis at the end of 8 weeks after STZ-induced diabetes. In order to validate the target genes in the diabetic hearts, mRNA expression levels of selected genes, including RASA1, RAC1, TGFB3 and COL1A1, were determined by real-time RT-PCR. RASA1, also known as RasGAP, plays a role in the cardiac myocyte growth induced by hypertrophic stimuli (19). Sayed et al revealed that the up-regulation of miR-1 decreased RasGAP mRNA expression levels in a TAC-induced hypertrophy mouse model (7). In this study, RASA1 was predicted to be one of the target genes of miR-1. An the increase in RASA1 mRNA was observed in diabetic hearts with cardiac hypertrophy, which further suggests that RASA1 is related to hyperglycemia-induced cardiac hypertrophy. Rac1, a small G protein, is reported to be critical for the development of cardiac hypertrophy in response to Ang II (20). Rac1 was also found to induce myocardial remodeling and cardiac dysfunction in diabetic mice (2). In our study, we identified RAC1 as a target of miR-142-3p and miR-700. The high RAC1 mRNA levels indicated that miR-142-3p or miR-700 may mediate RAC1, regulating cardiac hypertrophy in diabetic hearts. TGFB3 has been revealed to be involved in cardiac fibroblast proliferation and collagen biosynthesis in rats (21). In the present study, the mRNA levels of TGFB3, targeted by miR-29a, were increased in the diabetic hearts with myocardial fibrosis, suggesting that miR-29a may regulate myocardial fibrosis via TGFB3 in these hearts. COL1A1, the major fibrillar collagen produced by fibroblasts, acts as a regulator of cardiac fibrosis (22). The level of COL1A1 mRNA expression was increased in heart tissue showing myocardial fibrosis in diabetic mice. COL1A1 was determined to be a target of miR-29a and miR-705, which may be involved in myocardial fibrosis in diabetic cardiomyopathy. Although the expression of the remaining 188 target genes was not verified in our study, the majority have been noted to be involved in cardiac disease. For example, SMAD3, SMAD7, IGF1, CDC42, CCND1, COL3A1 and MAP3K7 have been reported to be involved in cardiac hypertrophy or myocardial fibrosis in vivo and in vitro (23-26).

To further examine the function of differentially expressed miRNAs, the 192 target genes were carefully annotated by GO analysis. Of five highly significant GO terms, three have already been reported to be involved in heart disease. Insulin-like growth factor-1 receptor (IGF1R) signaling is required for normal cardiac metabolism and cardiac function (27). Previous studies have also indicated that cardiac overexpression of IGF-1R prevented diabetes-induced cardiac hypertrophy, cardiac fibrosis and diastolic dysfunction in diabetic mice $(28,29)$. A number of studies have implicated JNKs in the regulation of cardiac hypertrophy in vitro and in vivo. JNK is activated in Ras-induced hypertrophied transgenic hearts, and specific activation of the JNK pathway in cardiac myocytes is sufficient to induce characteristic features of hypertrophy (30). The MAPKKK cascades lie downstream of numerous signaling pathways. It has been reported that MAPKKK may be involved in stretch-induced hypertrophic responses in neonatal rat cardiac myocytes (31). Although in our study other enriched GO terms were not found to be significant, most have already been reported to participate in cardiac hypertrophy or myocardial fibrosis in non-diabetic heart tissue and the diabetic heart. For example, Ras protein signal transduction and small guanosine triphosphatase (GTPase)-mediated signal transduction transmit a wide variety of extracellular signals to effector molecules within cells. Experimental and clinical evidence has revealed that RAS and small GTPase contribute to the development of left ventricular hypertrophy in hypertension $(32,33)$. Activation of Wnt signaling is sufficient and critical for the induction of myocardial hypertrophy and cardiomyopathy in transgenic mice characterized by significant cardiac hypertrophy (34). Transforming growth factor $\beta$ receptor signaling, the key pathway in fibrogenesis, has been noted to be essential for cardiac fibroblast proliferation in mice with myocardial fibrosis (35). Other enriched GO terms are fundamental to the biological processes of heart development and cardiac function. In light of these findings, it is possible that the target genes contribute to various biological processes involved in the pathogenesis of cardiac hypertrophy and myocardial fibrosis in diabetic cardiomyopathy.

In the present study, we determined the expression profile of microRNAs in diabetic cardiomyopathy, and discussed the targets and biological roles of these microRNAs. The findings established expression of several novel miRNAs, and suggest that miRNAs are differentially expressed between diabetic and non-diabetic hearts. Clearly, the identification of the regulatory targets of the miRNAs and the elucidation of their molecular function will aid in the determination of their biological functions.

\section{Acknowledgements}

This study was supported by the Shanghai Pujiang Program (no. 09PJ480400).

\section{References}

1. Boudina S and Abel ED: Diabetic cardiomyopathy revisited. Circulation 115: 3213-3223, 2007.

2. Li J, Zhu H, Shen E, Wan L, Arnold JM and Peng T: Deficiency of racl blocks NADPH oxidase activation, inhibits endoplasmic reticulum stress, and reduces myocardial remodeling in a mouse model of type 1 diabetes. Diabetes 59: 2033-2042, 2010.

3. Bartel DP: MicroRNAs: target recognition and regulatory functions. Cell 136: 215-233, 2009 .

4. Callis TE and Wang DZ: Taking microRNAs to heart. Trends Mol Med 14: 254-260, 2008.

5. Wang Z, Luo X, Lu Y and Yang B: miRNAs at the heart of the matter. J Mol Med 86: 771-783, 2008.

6. Scalbert E and Bril A: Implication of microRNAs in the cardiovascular system. Curr Opin Pharmacol 8: 181-188, 2008.

7. Sayed D, Hong C, Chen IY, Lypowy J and Abdellatif M: MicroRNAs play an essential role in the development of cardiac hypertrophy. Circ Res 100: 416-424, 2007.

8. Shen E, Diao X, Wei C, Wu Z, Zhang L and Hu B: MicroRNAs target gene and signaling pathway by bioinformatics analysis in the cardiac hypertrophy. Biochem Biophys Res Commun 397: 380-385, 2010.

9. Kolfschoten IG, Roggli E, Nesca V and Regazzi R: Role and therapeutic potential of microRNAs in diabetes. Diabetes Obes Metab 11 (Suppl 4): 118-129, 2009.

10. Gene Ontology Consortium: The Gene Ontology (GO) project in 2006. Nucleic Acids Res 34: D322-D326, 2006.

11. Barringhaus KG and Zamore PD: MicroRNAs: regulating a change of heart. Circulation 119: 2217-2224, 2009. 
12. Feng B, Chen S, George B, Feng Q and Chakrabarti S: miR133a regulates cardiomyocyte hypertrophy in diabetes. Diabetes Metab Res Rev 26: 40-49, 2010.

13. Carè A, Catalucci D, Felicetti F, et al: MicroRNA-133 controls cardiac hypertrophy. Nat Med 13: 613-618, 2007.

14. Callis TE, Pandya K, Seok HY, et al: MicroRNA-208a is a regulator of cardiac hypertrophy and conduction in mice. J Clin Invest 119: 2772-2786, 2009.

15. Van Rooij E, Sutherland LB, Liu N, et al: A signature pattern of stress-responsive microRNAs that can evoke cardiac hypertrophy and heart failure. Proc Natl Acad Sci USA 103: 18255-18260, 2006.

16. Cheng Y, Ji R, Yue J, et al: MicroRNAs are aberrantly expressed in hypertrophic heart: do they play a role in cardiac hypertrophy? Am J Pathol 170: 1831-1840, 2007.

17. Tatsuguchi M, Seok HY, Callis TE, et al: Expression of microRNAs is dynamically regulated during cardiomyocyte hypertrophy. J Mol Cell Cardiol 42: 1137-1141, 2007.

18. Bostjancic E, Zidar N and Glavac D: MicroRNA microarray expression profiling in human myocardial infarction. Dis Markers 27: 255-268, 2009.

19. Lypowy J, Chen IY and Abdellatif M: An alliance between Ras GTPase-activating protein, filamin C, and Ras GTPaseactivating protein SH3 domain-binding protein regulates myocyte growth. J Biol Chem 280: 25717-25728, 2005.

20. Satoh M, Ogita H, Takeshita K, Mukai Y, Kwiatkowski DJ and Liao JK: Requirement of Racl in the development of cardiac hypertrophy. Proc Natl Acad Sci USA 103: 7432-7437, 2006.

21. Abdelaziz N, Colombo F, Mercier I and Calderone A: Nitric oxide attenuates the expression of transforming growth factorbeta(3) mRNA in rat cardiac fibroblasts via destabilization. Hypertension 38: 261-266, 2001

22. Zhao HP, Lu D, Zhang W, et al: Protective action of tetramethylpyrazine phosphate against dilated cardiomyopathy in $\mathrm{cTnT}(\mathrm{R} 141 \mathrm{~W})$ transgenic mice. Acta Pharmacol Sin 31: 281-288, 2010.

23. Bhavsar PK, Brand NJ, Felkin LE, et al: Clenbuterol induces cardiac myocyte hypertrophy via paracrine signalling and fibroblast-derived IGF-1. J Cardiovasc Transl Res 3: 688-695, 2010.

24. Maillet M, Lynch JM, Sanna B, York AJ, Zheng Y and Molkentin JD: Cdc42 is an antihypertrophic molecular switch in the mouse heart. J Clin Invest 119: 3079-3088, 2009.
25. Huang XR, Chung AC, Yang F, et al: Smad3 mediates cardiac inflammation and fibrosis in angiotensin II-induced hypertensive cardiac remodeling. Hypertension 55: 1165-1171, 2010.

26. Wang B, Hao J, Jones SC, Yee MS, Roth JC and Dixon IM: Decreased Smad 7 expression contributes to cardiac fibrosis in the infarcted rat heart. Am J Physiol Heart Circ Physiol 282: H1685-H1696, 2002.

27. Laustsen PG, Russell SJ, Cui L, et al: Essential role of insulin and insulin-like growth factor 1 receptor signaling in cardiac development and function. Mol Cell Biol 27: 1649-1664, 2007.

28. Huynh K, McMullen JR, Julius TL, et al: Cardiac-specific IGF-1 receptor transgenic expression protects against cardiac fibrosis and diastolic dysfunction in a mouse model of diabetic cardiomyopathy. Diabetes 59: 1512-1520, 2010.

29. Schmidt B, Tilz RR, Neven K, Julian Chun KR, Furnkranz A and Ouyang F: Remote robotic navigation and electroanatomical mapping for ablation of atrial fibrillation: considerations for navigation and impact on procedural outcome. Circ Arrhythm Electrophysiol 2: 120-128, 2009.

30. Wang Y, Su B, Sah VP, Brown JH, Han J and Chien KR: Cardiac hypertrophy induced by mitogen-activated protein kinase kinase 7, a specific activator for c-Jun NH2-terminal kinase in ventricular muscle cells. J Biol Chem 273: 5423-5426, 1998.

31. Yamazaki T, Komuro I, Kudoh S, et al: Mechanical stress activates protein kinase cascade of phosphorylation in neonatal rat cardiac myocytes. J Clin Invest 96: 438-446, 1995.

32. Mathew J, Sleight P, Lonn E, et al: Reduction of cardiovascular risk by regression of electrocardiographic markers of left ventricular hypertrophy by the angiotensin-converting enzyme inhibitor ramipril. Circulation 104: 1615-1621, 2001

33. Kacimi R and Gerdes AM: Alterations in G protein and MAP kinase signaling pathways during cardiac remodeling in hypertension and heart failure. Hypertension 41: 968-977, 2003.

34. Malekar P, Hagenmueller M, Anyanwu A, et al: Wnt signaling is critical for maladaptive cardiac hypertrophy and accelerates myocardial remodeling. Hypertension 55: 939-945, 2010.

35. Sakata Y, Chancey AL, Divakaran VG, Sekiguchi K, Sivasubramanian N and Mann DL: Transforming growth factorbeta receptor antagonism attenuates myocardial fibrosis in mice with cardiac-restricted overexpression of tumor necrosis factor. Basic Res Cardiol 103: 60-68, 2008. 